Vietnam Journal of Mechanics, VAST, Vol.32, No. 3 (2010), pp. $167-181$

\title{
ON STABILIZATION OF THE NODE-BASED SMOOTHED FINITE ELEMENT METHOD FOR FREE VIBRATION PROBLEMS
}

\author{
Bui Xuan Thang ${ }^{1}$, Nguyen Xuan Hung ${ }^{1,2}$, Ngo Thanh Phong ${ }^{1}$ \\ ${ }^{1}$ University of Science - VNU - HCM \\ ${ }^{2}$ Ton Duc Thang University HCM
}

\begin{abstract}
The node-based smoothed finite element method (NS-FEM) has been recently proposed by Liu et al to enhance the computational effect for solid mechanics problems. However, it is evident that the NS-FEM behaves "overly-soft" and so it may lead to instability for dynamic problems. The instability can be clearly shown as spurious non-zero energy modes in free vibration analysis. In this paper, we present a stabilization of the node-based smoothed finite element method (SN-FEM) that is stable (no spurious non-zero energy modes) and more effective than the standard finite element method (FEM). Three numerical illustrations are given to evince the high reliability of the proposed formulation.
\end{abstract}

\section{INTRODUCTION}

Chen et al. [2] proposed the strain smoothing technique to achieve a stabilization in the nodal integrated meshfree methods. Then it is applied to the natural element method in [18]. This technique has been developed into a generalized smoothing technique by Liu et al. [5]. The generalized smoothing technique allows displacement functions to be discontinuous and forms the theoretical foundation for the linear conforming point interpolation method (LC-PIM) [9]. In addition, Liu et al. also introduced the linearly conforming radial point interpolation method (LC-RPIM) [4], the element-based smoothed finite element method (SFEM) [3, 10, 11, 14], the edge-based smoothed finite element method (ES-FEM) [6], and the node-based smoothed finite element method (NS-FEM) [8]. Concerning on the NS-FEM, the domain discretization is still based on element as same as the standard FEM. However, the formulation of the system stiffness matrix is appreciated by smoothing domain associated with nodes of elements. Four following properties of the NS-FEM were demonstrated by the numerical results: 1) it produces an upper bound (for problems driven by the applied forces) of the exact strain energy solution when meshes are reasonably fine; 2) it is free of volumetric locking; 3 ) the polygonal elements with an arbitrary number of sides can be used in the NS-FEM; 4) it doesn't need to use mapping or coordinate transformation in the NS-FEM whose element is allowed to be of arbitrary shape. We can apply easily the NS-FEM to triangular, 4-node quadrilateral and even nsided polygonal elements. Even the problem domain in the NS-FEM can be discretized 
into severely distorted elements. These features have been demonstrated numerically in [7].

Nevertheless, it is found that the NS-FEM behaves "overly-sof", that is contrast with the compatible FEM known "overly-stiff". The overly-soft behaviour makes the NSFEM be instable as the same way of the nodal integration methods $[16,15,12]$. This instability is clearly shown as spurious non-zero energy modes in free vibration problems.

In the other aspect of the development of numerical techniques, Puso and Solberg et al. [16] have formulated a stabilized nodally integrated linear tetrahedral to stabilize dynamics problems and to provide an effective tool for plasticity, nearly incompressible materials and acute bending problems.

In this paper, a stabilization procedure for remedying temporal instability of the node-based smoothed finite element method is presented for free vibration analysis of two-dimensional solid mechanics problems. The idea is to combine a stabilized nodally integrated technique into the existing NS-FEM using four-node quadrilateral elements. Several numerical examples are then given to show high reliability of proposed formulation.

\section{DISCRETE THE GOVERNING EQUATIONS}

Consider a deformable $2 \mathrm{D}$ body solid domain $\Omega$ moving in $\mathbb{R}^{2}$ subjected to body forces $\mathbf{b}$, external applied tractions $\mathbf{t}$ on boundary $\Gamma_{t}$ and displacement boundary conditions $\mathbf{u}=\overline{\mathbf{u}}$ on $\Gamma_{u}$. The principle of virtual work for the dynamic problems including the inertial and damping forces is that [6]

$$
\int_{\Omega} \delta \varepsilon^{T} \mathbf{D} \varepsilon \mathrm{d} \Omega-\int_{\Omega} \delta \mathbf{u}^{T}(\mathbf{b}-\rho \ddot{\mathbf{u}}-c \dot{\mathbf{u}}) \mathrm{d} \Omega-\int_{\Gamma_{t}} \delta \mathbf{u}^{T} \mathbf{t} \mathrm{d} \Gamma=0
$$

where $\mathbf{D}$ is a matrix of material constants that is symmetric positive definite, $\delta \varepsilon$ and $\delta \mathbf{u}$ are virtually compatible strains and displacements, respectively.

In FEM, the domain $\Omega$ is discretized into $N_{e}$ elements which of each element has $N P$ nodes variables. The virtual displacements and strains within any element can be written as

$$
\begin{aligned}
\delta \mathbf{u}_{h}^{e} & =\sum_{I=1}^{N P} \mathbf{N}_{I}(\mathbf{x}) \delta \mathbf{d}_{I}(t), & \mathbf{u}_{h}^{e} & =\sum_{I=1}^{N P} \mathbf{N}_{I}(\mathbf{x}) \mathbf{d}_{I}(t) \\
\delta \varepsilon_{h}^{e} & =\sum_{I=1}^{N P} \mathbf{B}_{I}(\mathbf{x}) \delta \mathbf{d}_{I}(t), & \varepsilon_{h}^{e} & =\sum_{I=1}^{N P} \mathbf{B}_{I}(\mathbf{x}) \mathbf{d}_{I}(t)
\end{aligned}
$$

where $\mathbf{d}_{I}=\left[\begin{array}{ll}u_{I} & v_{I}\end{array}\right]$ is the nodal displacement vector, $\mathbf{N}_{I}(\mathbf{x})$ and $\mathbf{b}_{I}$ are a shape function matrix and the standard displacement gradient matrix associated to node $I$, respectively. In 2D linear elastic problems, we have

$$
\mathbf{B}_{I}=\nabla_{s} N_{I}(x)=\left[\begin{array}{cc}
N_{I, x} & 0 \\
0 & N_{I, y} \\
N_{I, y} & N_{I, x}
\end{array}\right]
$$


Substituting (2), (3) into (1), it leads to

$\int_{\Omega} \delta \mathbf{d}^{T} \mathbf{B}^{T} \mathbf{D B d} \mathrm{d} \Omega-\int_{\Omega} \delta \mathbf{d}^{T} \mathbf{N}^{T}(\mathbf{b}-\rho \mathbf{N} \ddot{\mathbf{d}}-c \mathbf{N} \dot{\mathbf{d}}) \mathrm{d} \Omega-\int_{\Gamma_{t}} \delta \mathbf{d}^{T} \mathbf{N}^{T} \mathbf{t} \mathrm{d} \Gamma=0$

Since $\delta \mathbf{d}$ are any arbitrary virtual displacements, we obtain

$$
\int_{\Omega} \mathbf{B}^{T} \mathbf{D B} \mathrm{d} \Omega-\int_{\Omega} \mathbf{N}^{T}(\mathbf{b}-\rho \mathbf{N} \ddot{\mathbf{d}}-c \mathbf{N} \dot{\mathbf{d}}) \mathrm{d} \Omega-\int_{\Gamma_{t}} \mathbf{N}^{T} \mathbf{t} \mathrm{d} \Gamma=0
$$

The result discrete governing equation can be written as

$$
\mathbf{M} \ddot{\mathbf{d}}+\mathbf{C} \dot{\mathbf{d}}+\mathbf{K d}=\mathbf{f}
$$

where

$$
\begin{gathered}
\mathbf{M}=\int_{\Omega} \mathbf{N}^{T} \rho \mathbf{N} \mathrm{d} \Omega \\
\mathbf{C}=\int_{\Omega} \mathbf{N}^{T} c \mathbf{N} \mathrm{d} \Omega \\
\mathbf{K}=\int_{\Omega} \mathbf{B}^{T} \mathbf{D} \mathbf{B} \mathrm{d} \Omega \\
\mathbf{f}=\int_{\Omega} \mathbf{N}^{T} \mathbf{B} \mathrm{d} \Omega+\int_{\Gamma_{t}} \mathbf{N}^{T} \mathbf{t} \mathrm{d} \Gamma
\end{gathered}
$$

and $\mathbf{d}$ is the vector of general nodal displacements.

\section{THE STABILIZATION OF THE NODE-BASED SMOOTHED FOUR-NODE QUADRILATERAL ELEMENT (SN-FEM-Q4)}

The formulation given above is the standard form of the FEM. The SN-FEM uses a mesh of elements as the same way of the standard FEM. When quadrilateral elements are used, the shape functions used in the SN-FEM-Q4 can be also exploited from those of the FEM-Q4.

In the SN-FEM, however, the compatible strains are replaced by strains "smoothed" over local smoothing domains, and naturally the integration related to the stiffness matrix $\overline{\mathbf{K}}$ is now not only based on elements but also smoothing domains. These local smoothing domains are constructed based on nodes of the elements. In the SN-FEM setting, the problem domain is additionally divided into smoothing domain associated with nodes in the same way of the NS-FEM in [8]. The domain discretization is such that $\Omega=$ $\Omega^{(1)} \cup \Omega^{(2)} \cup \ldots \cup \Omega^{\left(N_{s}\right)}$ and $\Omega^{(i)} \cap \Omega^{(j)}=\emptyset, i \neq j$, where $N_{s}$ is the total number of nodes in the entire domain. For four-node quadrilateral elements, a cell $\Omega^{(k)}$ associated with the node $k$ is created by four sub-cells as shown in Fig. 1. As a result, each four-node quadrilateral element is divided into four sub-cells and each sub-cell is attached with the nearest field node.

In this paper, we justify the stiffness matrix of the existing NS-FEM by adding a stabilized term using the standard FEM stiffness matrix. The formulation of the stiffness matrix $\mathbf{K}$ of SN-FEM is now given as [16]:

$$
\overline{\mathbf{K}}=\mathbf{K}_{\text {nodal }}+\mathbf{K}_{\text {elem }}
$$


where $\mathbf{K}_{\text {nodal }}$ is the stiffness matrix associated with the node $k$

$$
\mathbf{K}_{\text {nodal }}=\sum_{k=1}^{N_{s}} \int_{\Omega^{(k)}} \overline{\mathbf{B}}\left(\mathbf{x}_{k}\right)^{T}(\mathbf{D}-\alpha \overline{\mathbf{D}}) \overline{\mathbf{B}}\left(\mathbf{x}_{k}\right) \mathrm{d} \Omega
$$

and $\mathbf{K}_{\text {elem }}$ is the typical quadrilateral stiffness matrix

$$
\mathbf{K}_{\text {elem }}=\sum_{e=1}^{N_{e}} \alpha \int_{\Omega^{e}} \mathbf{B}^{T} \overline{\mathbf{D}} \mathbf{B} \mathrm{d} \Omega
$$

where $\alpha$ is a stabilization parameter discussed in [16], $\overline{\mathbf{D}}$ could be an alternative material matrix, $\overline{\mathbf{B}}\left(\mathbf{x}_{k}\right)$ is the gradient of displacement based on nodes of elements, and $\Omega^{e}$ is the domain of an element. The material matrices $\mathbf{D}$ and $\overline{\mathbf{D}}$ are given in terms of Lamé parameters $\lambda$ and $\mu$

$$
\mathbf{D}=\left[\begin{array}{ccc}
\lambda+2 \mu & \lambda & 0 \\
\lambda & \lambda+2 \mu & 0 \\
0 & 0 & \mu
\end{array}\right], \overline{\mathbf{D}}=\left[\begin{array}{ccc}
\bar{\lambda}+2 \bar{\mu} & \bar{\lambda} & 0 \\
\bar{\lambda} & \bar{\lambda}+2 \bar{\mu} & 0 \\
0 & 0 & \bar{\mu}
\end{array}\right]
$$

Lamé parameters in the plane stress and plane strain, respectively, are:

$$
\lambda=\frac{E \mu}{\left(1-\mu^{2}\right)}, \quad \lambda=\frac{E \mu}{(1+\mu)(1-2 \mu)},
$$

where $E$ is Young's Modulus and $\mu$ is Poisson's ratio. $\mathbf{D}=\overline{\mathbf{D}}$ when material is compressible. If material is nearly incompressible with Poisson's ratio, $\mu=0.4999$ for example, $\overline{\mathbf{D}}$ is different from $\mathbf{D}$ by choosing the effective Lamé parameters $\bar{\mu}, \bar{\lambda}$. A practical choice for $\bar{\mu}, \bar{\lambda}$ is given in Section 4. The gradient of displacement $\overline{\mathbf{B}}\left(\mathbf{x}_{k}\right)$ is formulated below. In general, the stabilization parameter $\alpha$ depends on the element and nodes of the element, but in this paper we choose $\alpha$ uniformly.

The gradient of displacement $\overline{\mathbf{B}}^{(k)}$ was formulated in [8] by introducing the nodebased strain smoothing operation:

$$
\bar{\varepsilon}^{(k)}=\int_{\Omega^{(k)}} \varepsilon(\mathbf{x}) \Phi^{(k)}(\mathbf{x}) \mathrm{d} \Omega
$$

where $\Phi^{(k)}(\mathbf{x})$ is a step function given by

$$
\Phi^{(k)}(\mathbf{x})= \begin{cases}1 / A^{(k)} & \mathbf{x} \in \Omega^{(k)} \\ 0 & \mathbf{x} \notin \Omega^{(k)}\end{cases}
$$

where $A^{(k)}$ is the area of the smoothing domain $\Omega^{(k)}$.

Substituting Equations (18) into Equations (17), we get the smoothed strain

$$
\bar{\varepsilon}^{(k)}=\frac{1}{A^{(k)}} \int_{\Gamma^{(k)}} \mathbf{u}(\mathbf{x}) \mathbf{n}^{(k)}(\mathbf{x}) \mathrm{d} \Gamma
$$




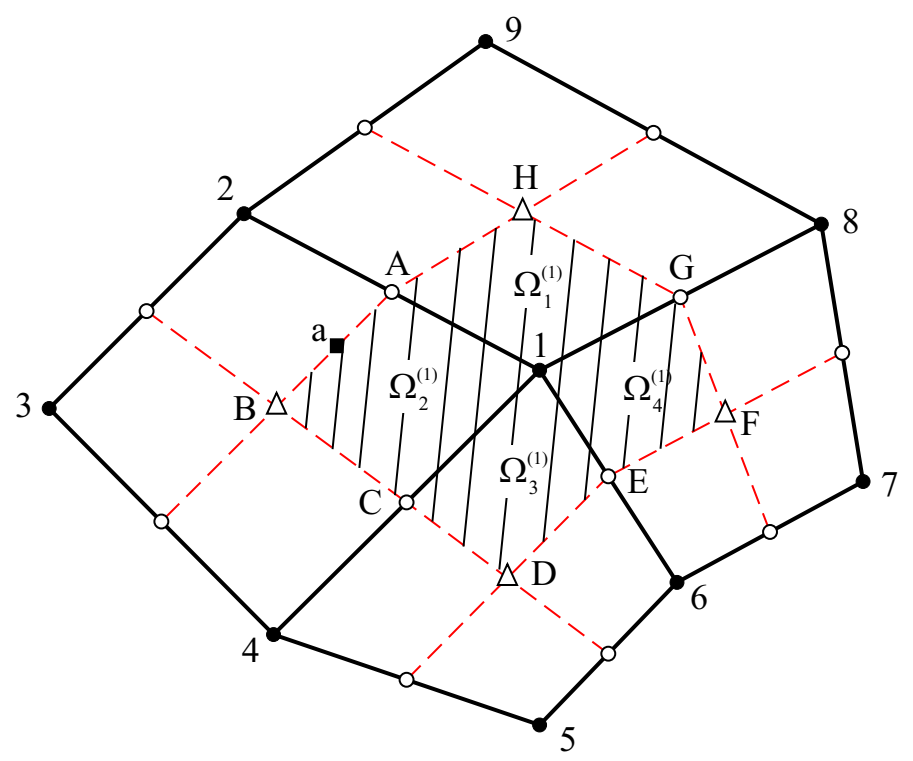

Fig. 1. Smoothing domain $\Omega^{(1)}$ associated with node 1 that consists of subdomains: The symbols $(\bullet),(\circ)$, and $(\triangle)$ denote the nodal field, the mid-edge point and the intersection point of two bi-medians of element, respectively.

where $\Gamma^{(k)}$ is the boundary of domain $\Omega^{(k)}$ as shown in Fig. 1, and $\mathbf{n}^{(k)}(\mathbf{x})$ is the outward normal vector matrix of the boundary $\Gamma^{(k)}$

$$
\mathbf{n}^{(k)}(\mathbf{x})=\left[\begin{array}{ll}
n_{x}^{(k)} & 0 \\
0 & n_{y}^{(k)} \\
n_{y}^{(k)} & n_{x}^{(k)}
\end{array}\right]
$$

Substituting Equation (2) into Equation (19), the formulation of the smoothed strain at node $k$ become the following matrix form of nodal displacements

$$
\bar{\varepsilon}^{(k)}=\sum_{I \in N^{(k)}} \overline{\mathbf{B}}_{I}\left(\mathbf{x}_{k}\right) \mathbf{d}_{I}
$$

where $N^{(k)}$ is the number of nodes connected to node $k$ directly and $\overline{\mathbf{B}}_{I}\left(\mathbf{x}_{k}\right)$ is the smoothed gradient matrix on the cell $\Omega^{(k)}$

$$
\overline{\mathbf{B}}_{I}\left(\mathbf{x}_{k}\right)=\left[\begin{array}{cc}
\bar{b}_{I x}\left(\mathbf{x}_{k}\right) & 0 \\
0 & \bar{b}_{I y}\left(\mathbf{x}_{k}\right) \\
\bar{b}_{I y}\left(\mathbf{x}_{k}\right) & \bar{b}_{I x}\left(\mathbf{x}_{k}\right)
\end{array}\right]
$$

where

$$
\bar{b}_{I h}\left(\mathbf{x}_{k}\right)=\frac{1}{A^{(k)}} \int_{\Gamma^{(k)}} N_{I}(\mathbf{x}) n_{h}^{(k)}(\mathbf{x}) \mathrm{d} \Gamma, \quad(h=x, y)
$$

Because of a linear compatible displacement field along boundary $\Gamma^{(k)}$, one Gaussian point is sufficient to calculate line integration along each segment $\Gamma_{b}^{(k)}$ of the boundary. 
Equation( 23) can be calculated numerically by its algebraic form

$$
\bar{b}_{I h}\left(\mathbf{x}_{k}\right)=\frac{1}{A^{(k)}} \sum_{b=1}^{n b} N_{I}\left(\mathbf{x}_{b}^{G P}\right) n_{b h}^{(k)} l_{b}^{(k)}, \quad(h=x, y)
$$

where $n b$ is the total number of the edges of $\Gamma^{(k)}, \mathbf{x}_{b}^{G P}$ is Gaussian point. $\Gamma_{b}^{(k)}$ has length and outward unit normal are denoted as $l_{b}^{(k)}$ and $n_{b h}^{(k)}$, respectively. An assembly process of the global stiffness matrix $\mathbf{K}_{\text {nodal }}$ based on looping over nodes of elements. From the Equation (13), we find the formulation of the nodal stiffness matrix of any node $k$

$$
\begin{aligned}
\mathbf{K}_{\text {nodal }}^{(k)} & =\int_{\Omega^{(k)}} \overline{\mathbf{B}}\left(\mathbf{x}_{k}\right)^{T}(\mathbf{D}-\alpha \overline{\mathbf{D}}) \overline{\mathbf{B}}\left(\mathbf{x}_{k}\right) \mathrm{d} \Omega= \\
& =\frac{1}{A^{(k)}}\left(\int_{\Gamma^{(k)}} \mathbf{n}^{(k)} \mathbf{N} \mathrm{d} \Gamma\right)^{T}(\mathbf{D}-\alpha \overline{\mathbf{D}})\left(\int_{\Gamma^{(k)}} \mathbf{n}^{(k)} \mathbf{N} \mathrm{d} \Gamma\right)
\end{aligned}
$$

The force vector obtained in the SN-FEM is the same as that in the FEM.

\section{NUMERICAL RESULTS}

In this section, we show the performance of the SN-FEM-Q4 for free vibration analysis of 2D solid problems. For linear elasticity, SN-FEM-Q4 becomes to the standard FEM-Q4 when $\alpha=1$ and $\overline{\mathbf{D}}=\mathbf{D}$. In addition, as $\alpha=0$, SN-FEM-Q4 is identical to the node-based smoothed four-node quadrilateral element (the NS-FEM-Q4). In following examples, we choose the uniform value of stabilization parameter such that $\alpha=0.05$. A choice of stabilization matrix $\overline{\mathbf{D}}$ was discussed by Puso et al. [16]. It was chosen to minimize the volumetric locking effective while providing an optimal stabilization necessary. For elastic materials, these effective modulus based on the Lamé parameters $\lambda$ and $\mu$ are assigned as [16]

$$
\bar{\mu}=\mu \quad \text { and } \quad \bar{\lambda}=\min (\lambda, 25 \bar{\mu})
$$

Assumed without damping and forcing terms, Equation (7) now reduces to a homogeneous equation:

$$
\mathbf{M} \ddot{\mathbf{d}}+\overline{\mathbf{K}} \mathbf{d}=0
$$

A general solution of a homogeneous equation is

$$
\mathbf{d}=\overline{\mathbf{d}} \exp (i \omega t)
$$

where $t$ indicates time, $\overline{\mathbf{d}}$ is the eigenvector and $\omega$ is natural frequency. On its substitution into Equation (27), then solving the following eigenvalue equation to find the natural frequency $\omega$

$$
\left(-\omega^{2} \mathbf{M}+\overline{\mathbf{K}}\right) \overline{\mathbf{d}}=0 .
$$

In this paper, we used consistent mass to calculate the mass matrix. So that, the formulation of mass matrix for any element is

$$
\mathbf{M}_{e}=\int_{\Omega_{e}} \rho(\mathbf{N})^{T} \mathbf{N} \mathrm{d} \Omega .
$$




\subsection{A cantilever beam loaded at the free end: the convergence study}

In the first numerical problem, we suggest studying the rectangular cantilever beam subjected to the parabolic loading at the free end as shown in Fig. 2. The beam has a so smaller thickness than the length $L$ and height $D$, so the plane stress is valid. The analytical solution can be found in the text book by Timoshenko and Goodier [17]:

$$
\begin{gathered}
u_{x}=\frac{P y}{6 \bar{E} I}\left[(6 L-3 x) x+(2+\bar{\nu})\left(y^{2}-\frac{D^{2}}{4}\right)\right], \\
u_{y}=-\frac{P}{6 \bar{E} I}\left[3 \bar{\nu} y^{2}(L-x)+(4+5 \bar{\nu}) \frac{D^{2} x}{4}+(3 L-x) x^{2}\right],
\end{gathered}
$$

where $I$ is the moment of inertia for the beam with a rectangular cross section and unit thickness has formula $I=D^{3} / 12$ and

$$
\begin{gathered}
\bar{E}=E, \quad \bar{\nu}=\nu \quad \text { for plane stress } \\
\bar{E}=\frac{E}{1-\nu^{2}}, \quad \bar{\nu}=\frac{\nu}{1-\nu} \quad \text { for plane strain. }
\end{gathered}
$$

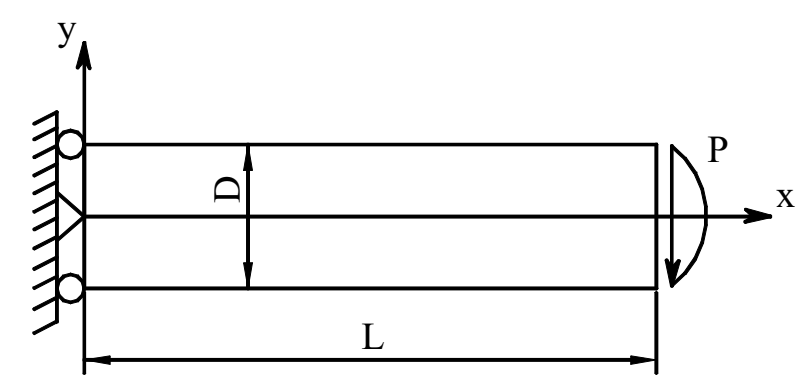

Fig. 2. The cantilever beam loaded at the free end

Stresses according to the displacements are

$$
\sigma_{x x}(x, y)=\frac{P(L-x) y}{I}, \sigma_{y y}(x, y)=0, \tau_{x y}(x, y)=-\frac{P}{2 I}\left(\frac{D^{2}}{4}-y^{2}\right) .
$$

The elastic material properties are following: $E=3.0 \times 10^{7} \mathrm{~N} / \mathrm{m}^{2}, \mu=0.3$. The related parameters are length $L=48 \mathrm{~m}$, height $D=12 \mathrm{~m}$, and loading $P=1000 \mathrm{~N}$. A mesh of quadrilateral elements is illustrated in Fig. 3.

Now we study the error in energy norm to make clear the statement that SN-FEMQ4 is more accurate than FEM-Q4. The error of energy norm is defined as

$$
\left\|\mathbf{u}-\mathbf{u}^{h}\right\|_{e}=\left[\sum_{i=1}^{N_{e}} \int_{\Omega^{e}}\left(\varepsilon-\varepsilon^{h}\right)^{T} \mathbf{D}\left(\varepsilon-\varepsilon^{h}\right) \mathrm{d} \Omega\right]^{1 / 2},
$$




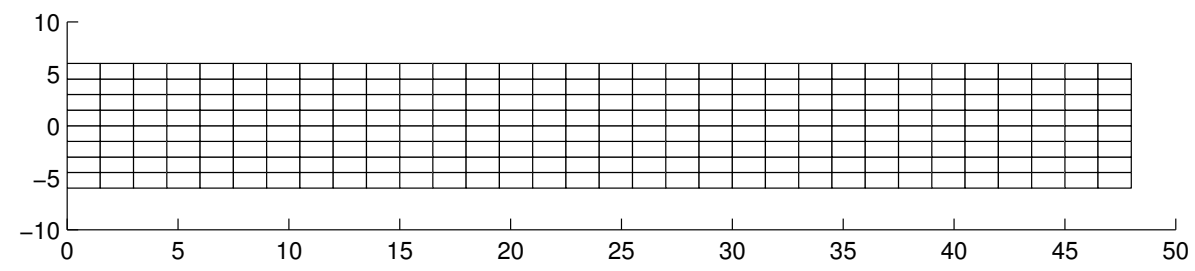

Fig. 3. The cantilever beam loaded at the free end

where $\mathbf{u}$ and $\varepsilon$ are the exact solution of the problem and $\mathbf{u}^{h}$ and $\varepsilon^{h}$ are the numerical solution of the displacement and strain of an element.

The convergence rate of the energy norm is evaluated by the "averaged" length of sides of element:

$$
h=\sqrt{\frac{A_{\Omega}}{N_{e}}}
$$

where $A_{\Omega}$ is an area of the problem domain. Fig. 4 shows the convergence of the error in energy norm for plane stress case. The same convergence rate of the original NS-FEMQ4 (1.34) and SN-FEM-Q4 (1.35) is obtained and is higher than that of the FEM-Q4 (0.996). The SN-FEM-Q4 produces the same accuracy the NS-FEM-Q4 while it shows better accuracy than the FEM-Q4.

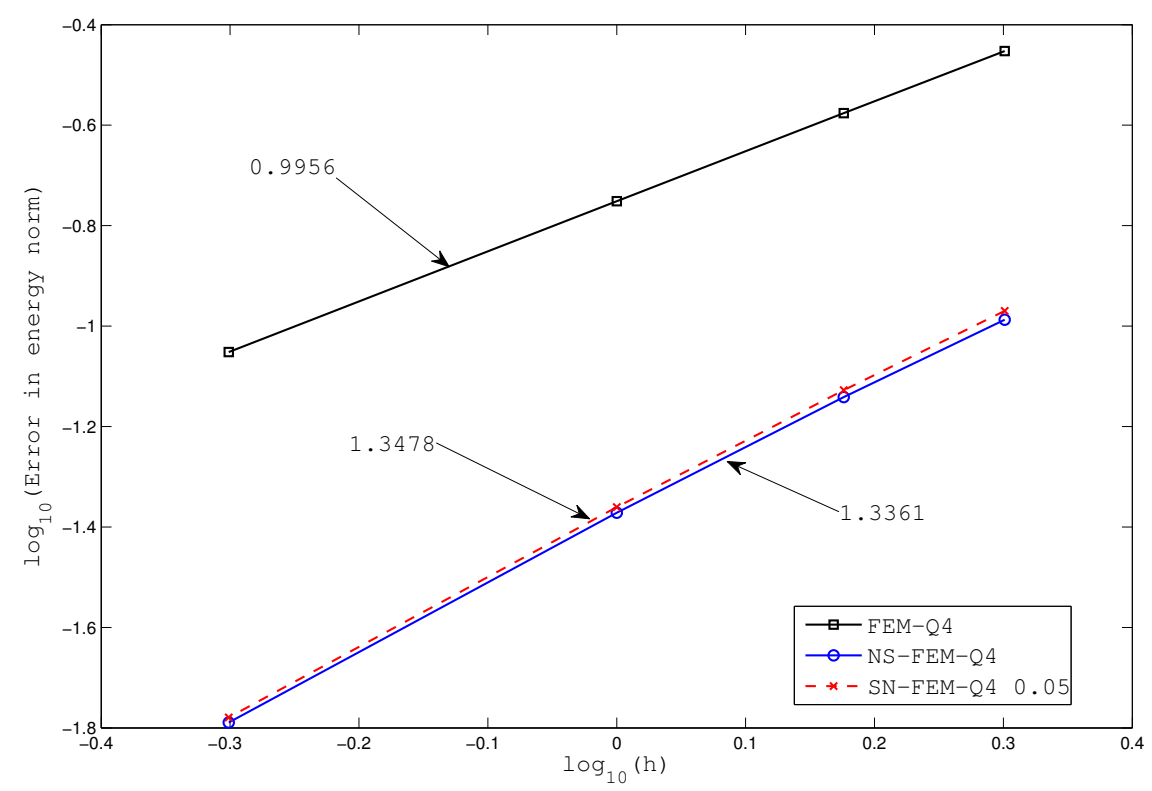

Fig. 4. Error in the energy for the cantilever beam of the SN-FEM-Q4 is compared with FEMQ4 and NSQ4 


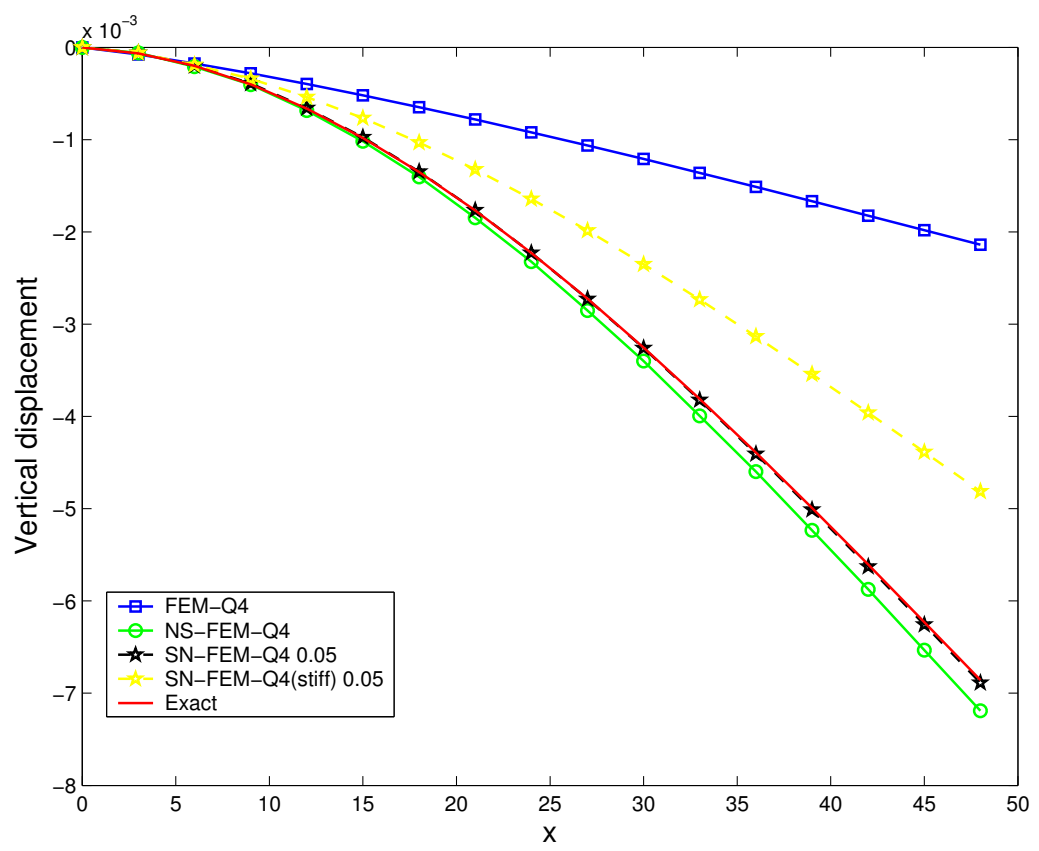

Fig. 5. The vertical displacement at neutralize axis $(y=0)$ of the cantilever beam. SN-FEM-Q4 shows very good agreement compared to analytical solution of incompressibility, $\mu=0.4999$

The displacement of nodes at some specific locations on the beam is plotted in Fig. 5 to illustrate results in the choice of the effective Lamé for incompressible cases $(\nu \simeq 0.5)$. For plane strain assumption in that case $(\nu \simeq 0.5)$, the appearance of volumetric locking makes the solution of FEM-Q4 be too stiff. The SN-FEM-Q4 (stiff) using the actual modulus $\lambda$ instead of effective modulus $\bar{\lambda}$ in Eq. (26) is also illustrated. It is observed that the SN-FEM-Q4 solution with $\alpha=0.05$ is closest to the exact one. In the next example, we show that the SN-FEM-Q4 is stable while the NS-FEM is unstable for free vibration analysis.

\subsection{Free vibration analysis of a cantilever beam}

A cantilever beam is studied with length $L=100 \mathrm{~mm}$, height $D=10 \mathrm{~mm}$, thickness $t=1 \mathrm{~mm}$, Young's Modulus $E=2.1 \times 10^{4} \mathrm{kgf} / \mathrm{mm}^{2}$, Poison's ratio $\nu=0.3$, mass density $\rho=8.0 \times 10^{-10} \mathrm{kgfs}^{2} / \mathrm{mm}^{4}$. A plane stress problem is considered.

Fig. 6 is evident that spurious modes appear without using a stabilized technique. It is clear that spurious modes are vanished after applying the stabilization formulation shown in Fig. 7. Table 1 illustrates frequencies of SN-FEM-Q4 compared to those of FEMQ4, NS-FEM-Q4, and NS-FEM-T3 [6] using the refined mesh. It can be seen that the SN-FEM-Q4 solution shows a good agreement compared to the reference value while NS-FEM-Q4 and NS-FEM-T3 are unstable with the appearance of spurious modes. 

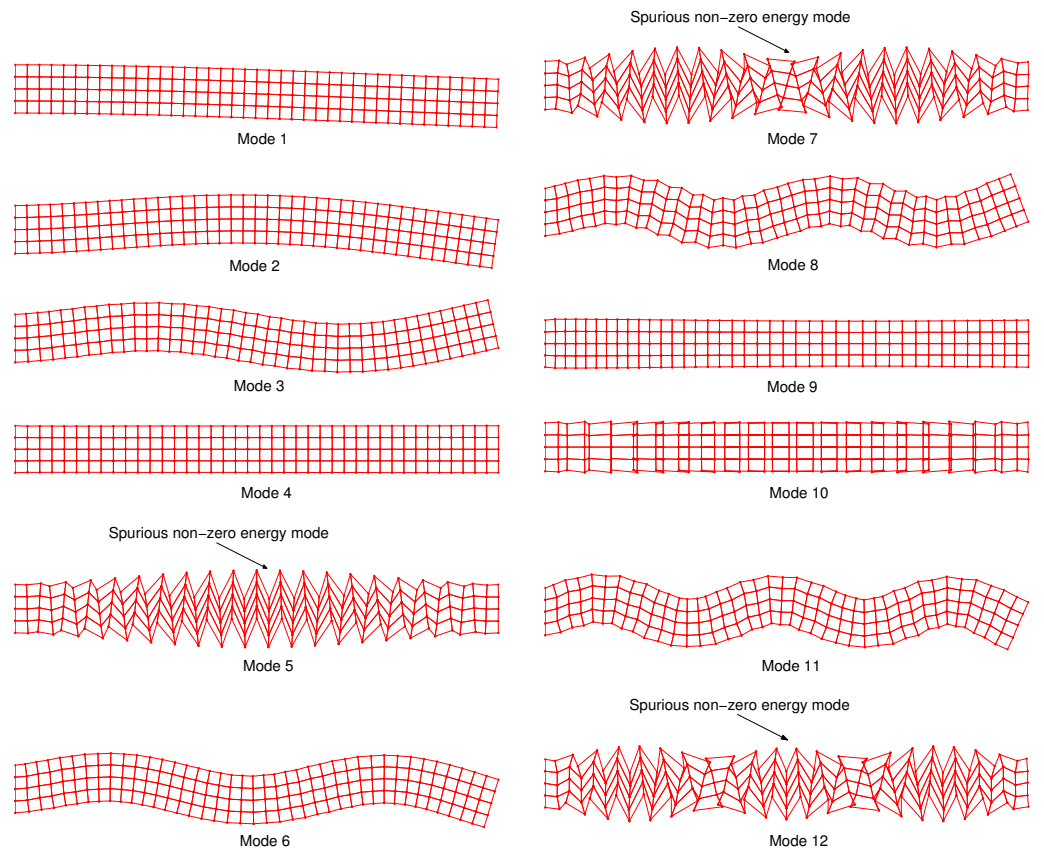

Fig. 6. First twelve modes of the cantilever beam by the NS-FEM-Q4: The presentation of spurious modes

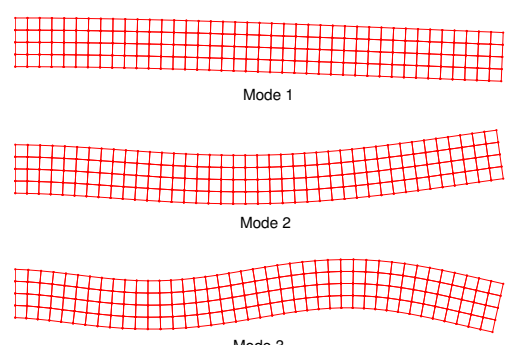

Mode 3

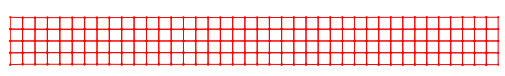

Mode 4

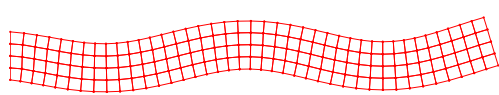

Mode 5

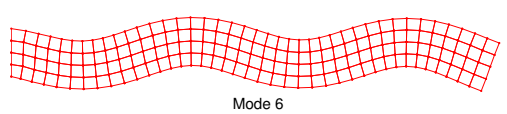

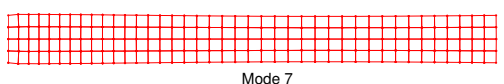
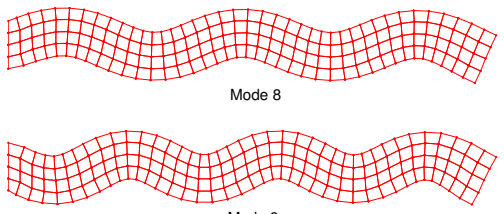

Mode 9

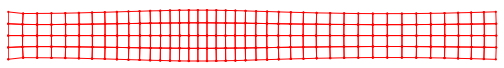

Mode 10
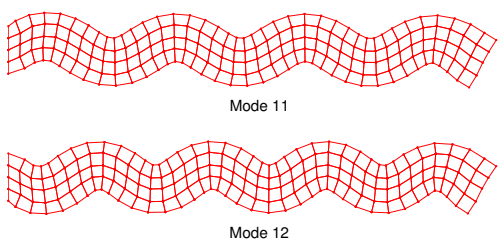

Fig. 7. First twelve modes of the cantilever beam by the SN-FEM-Q4: No presentation of spurious modes 
Table 1. First 12 natural frequencies $\left(\times 10^{4} \mathrm{~Hz}\right)$ of a cantilever beam

\begin{tabular}{|c|c|c|c|c|c|}
\hline No. of elements & No. of nodes & $\begin{array}{c}\text { NS-FEM-T3 } \\
\text { ref. }[6]\end{array}$ & NS-FEM-Q4 & SN-FEM-Q4 & $\begin{array}{l}\text { FEM-Q4 } \\
100 \times 10 \\
\end{array}$ \\
\hline \multirow[t]{12}{*}{$20 \times 2$} & \multirow[t]{12}{*}{63} & 0.0675 & 0.0755 & 0.0763 & 0.0824 \\
\hline & & 0.4032 & 0.4492 & 0.4593 & 0.4944 \\
\hline & & 1.0518 & $0.8048^{*}$ & 1.2102 & 1.2824 \\
\hline & & 1.2810 & 1.1651 & 1.2820 & 1.3022 \\
\hline & & $1.6467^{*}$ & 1.2812 & 2.1918 & 2.3663 \\
\hline & & 1.8786 & $1.8112^{*}$ & 3.3149 & 3.6085 \\
\hline & & $2.7823^{*}$ & 2.0685 & 3.8290 & 3.8442 \\
\hline & & 3.0926 & 2.5624 & 4.5002 & 4.9674 \\
\hline & & 3.6783 & $2.9194^{*}$ & 5.6847 & 6.3960 \\
\hline & & 3.8089 & 3.0579 & 6.3243 & 6.4023 \\
\hline & & $4.0543^{*}$ & 3.8078 & 6.8150 & 7.8853 \\
\hline & & $4.1605^{*}$ & $3.9820^{*}$ & 7.8353 & 8.9290 \\
\hline \multirow[t]{12}{*}{$40 \times 4$} & \multirow[t]{12}{*}{205} & 0.0778 & 0.0801 & 0.0802 & 0.0824 \\
\hline & & 0.4654 & 0.4780 & 0.4808 & 0.4944 \\
\hline & & 1.2199 & $0.8286^{*}$ & 1.2632 & 1.2824 \\
\hline & & 1.2818 & 1.2504 & 1.2821 & 1.3022 \\
\hline & & $1.6689^{*}$ & 1.2819 & 2.2873 & 2.3663 \\
\hline & & 2.2012 & $1.8866^{*}$ & 3.4732 & 3.6085 \\
\hline & & $3.2517^{*}$ & 2.2516 & 3.8400 & 3.8442 \\
\hline & & 3.3270 & 2.5697 & 4.7572 & 4.9674 \\
\hline & & 3.8344 & $3.1196^{*}$ & 6.0959 & 6.3960 \\
\hline & & 4.5248 & 3.3969 & 6.3781 & 6.4023 \\
\hline & & $4.6406^{*}$ & 3.8346 & 7.4584 & 7.8853 \\
\hline & & $5.3275^{*}$ & $4.4052^{*}$ & 8.8221 & 8.9290 \\
\hline
\end{tabular}

* Spurious non-zero energy modes

\subsection{Free vibration analysis of a shear wall}

In this example we analyze a shear wall with four openings as the Fig. 8. The numerical solutions solved by SN-FEM-Q4 are compared with solutions found in Brebbia [1]. A bottom edge of the wall is fully clamped. A plane stress problem is considered with $E=10,000, \nu=0.2, t=1.0$, and $\rho=1.0$.

Table 2. First 6 natural frequencies of a shear wall

\begin{tabular}{cccccc}
\hline $\begin{array}{c}\text { No. of } \\
\text { elements }\end{array}$ & $\begin{array}{c}\text { No. of } \\
\text { nodes }\end{array}$ & $\begin{array}{c}\text { NS-FEM-T3 } \\
\text { ref. [6] }\end{array}$ & NS-FEM-Q4 & SN-FEM-Q4 & $\begin{array}{c}\text { Reference } \\
\text { (Brebbia et al [1]) }\end{array}$ \\
\hline 476 & 559 & 1.8272 & 1.8721 & 1.9217 & 2.079 \\
& & 6.5113 & 6.6604 & 6.7438 & 7.181 \\
& & 7.5147 & 7.5220 & 7.5658 & 7.644 \\
& & 10.1828 & $10.5206^{\dagger}$ & 10.8449 & 11.833 \\
& & 13.7335 & $14.1997^{\dagger}$ & 14.4028 & 15.947 \\
\hline
\end{tabular}

$\dagger$ Spurious non-zero energy modes 


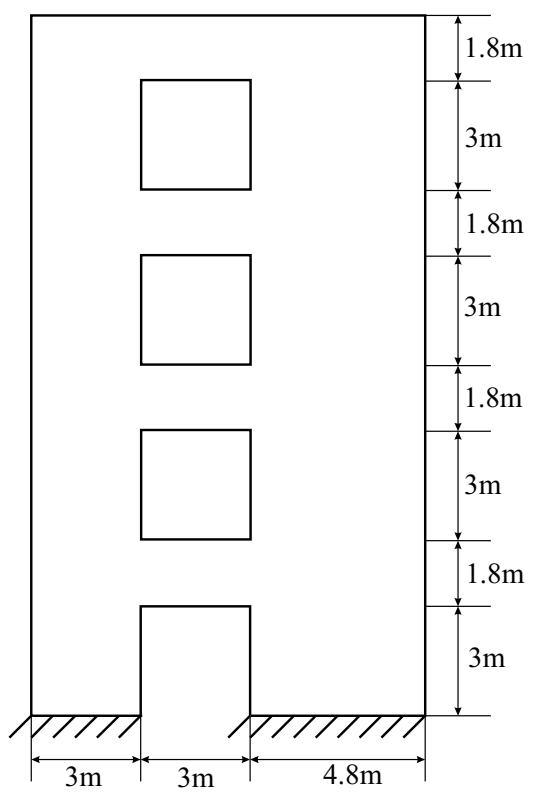

Fig. 8. A shear wall has four openings and a fully clamped bottom edge.

Fig. 9 shows the appearance of spurious modes when NS-FEM-Q4 is used. This problem can be solved when we use SN-FEM-Q4 as shown in Fig. 10. Table 2 illustrates first six natural frequencies of the shear wall. The SN-FEM-Q4 works well for this problem.
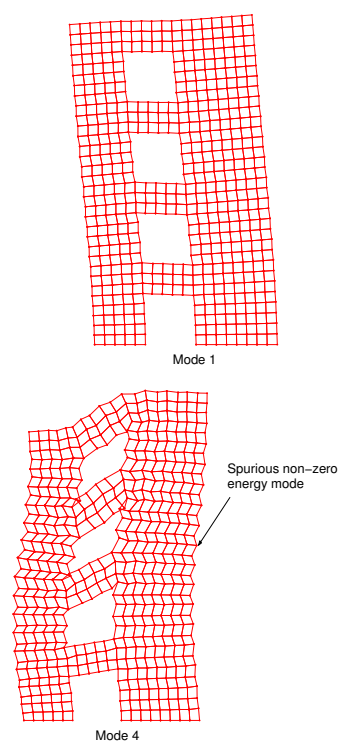

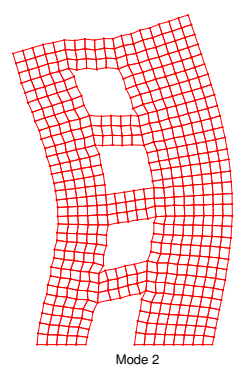

Mode 2

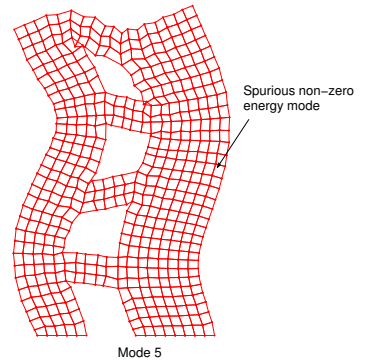

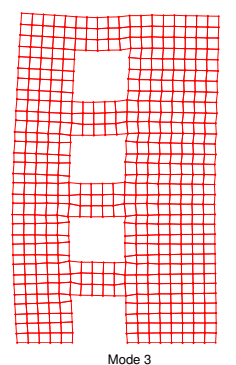

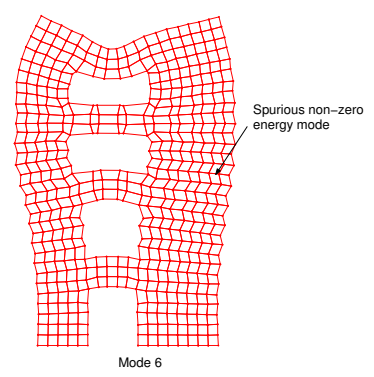

Fig. 9. First six modes of the shear wall by the NS-FEM: The presentation of spurious modes 

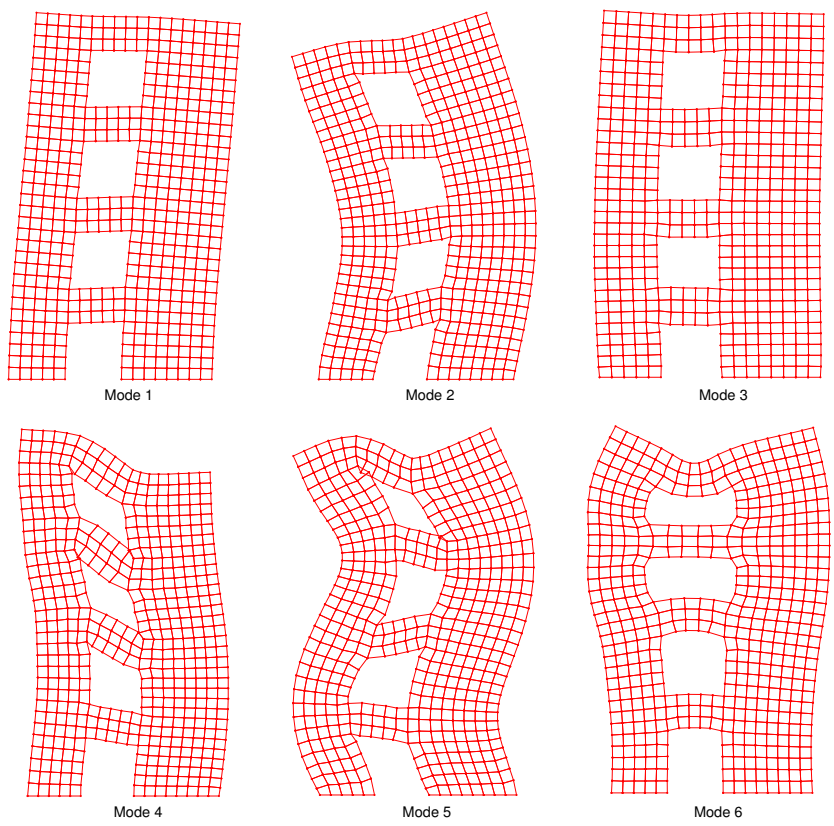

Fig. 10. First six modes of the shear wall by the SN-FEM-Q4: No presentation of spurious modes

\section{CONCLUSION}

In this paper, we have presented the SN-FEM-Q4 formulation and analyzed its effectiveness by illustrating several numerical examples. The results are compared with the original NS-FEM-Q4 and the standard FEM-Q4. It is observed that the SN-FEM-Q4 solution is more accurate than the FEM-Q4 element in energy norm and insensitive to volumetric locking. The SN-FEM-Q4 can produce super-convergence of stress solutions that is similar to the original NS-FEM-Q4 while SN-FEM-Q4 displacements are more accurate than NS-FEM-Q4 one. Moreover the SN-FEM-Q4 is stable in free vibration analysis (no spurious non-zero energy modes) with stabilization parameter $\alpha=0.05$ while the original NS-FEM is instable. As a result, the SN-FEM-Q4 inherits the advantages of the NS-FEM and provides temporal stability for solving the free vibration problems.

\section{REFERENCES}

[1] C. A. Brebbia, J. C. Telles and L. C. Wrobel, Boundar,y Element techniques, Springer, Berlin (1984).

[2] J. S. Chen, T. Wu, S. Yoon and Y. You, A stabilized conforming nodal integration for Galerkin mesh-free methods, International Journal for Numerical Methods in Engineering 50 (2001) 435-466.

[3] K.Y. Dai, G.R. Liu, and T.T. Nguyen, An n-sided polygonal smoothed finite element method (nSFEM) for solid mechanics, Finite elements in analysis and design 43 (2007) 847-860. 
[4] K.Y. Dai, M.T. Luan, W. Xue, G.R. Liu and Y. Li, A linearly conforming radial point interpolation method for solid mechanics problems, International Journal of Computational Methods 3(4) 401-428.

[5] G. R. Liu, A generalized gradient smoothing technique and the smoothed bilinear form for galerkin formulation of a wide class of computational methods, International Journal of Computational Methods 5(2) (2008) 199-236.

[6] G. R. Liu, T. Nguyen-Thoi, and K. Y. Lam, An edge-based smoothed finite element method (NS-FEM) for static, free and forced vibration analyses of solids, Journal of Sound and Vibration 320 (2009) 1100-1130.

[7] G. R. Liu, T. Nguyen-Thoi, H. Nguyen-Xuan, and K. Y. Lam, A node-based smoothed finite element method (NS-FEM) for upper bound solutions to solid mechanics problems, Comput. Struct. 87(1-2) (2009), 14-26.

[8] G. R. Liu, H. Nguyen-Xuan, and T. Nguyen-Thoi, A theoretical study on NS/ES-FEM: properties, accuracy and convergence rates, International Journal for Numerical Methods in Engineering (2010) accepted.

[9] G. R. Liu, G. Y. Zang, K. Y. Dai, Y. Y. Wang, Z. H. Zong, G. Y. Li, and X. Han, A linearly conforming point interpolation method (LC-PIM) for 2D solid mechanics problems, International Journal of Computational Methods 2(4) 645-665.

[10] G. R. Liu, K. Y. Dai, and T. T. Nguyen, A smoothed finite element for mechanics problems, Computational Mechanics 39 (2007) 859-877.

[11] G. R. Liu, T. T. Nguyen, K. Y. Dai, and K. Y. Lam, Theoretical aspects of the smoothed finite element method (SFEM), International Journal for Numerical Methods in Engineering 71 (2007) 902-930.

[12] Toshio Nagashima, Node-by-node meshless approach and its applications to structural analyses, International Journal for Numerical Methods in Engineering 46 (1999) 341-385.

[13] H. Nguyen-Xuan, S. Bordas and H. Nguyen-Dang, Smooth finite element methods: Convergence, accuracy and properties, International Journal for Numerical Methods in Engineering 74 (2008) 175-208.

[14] H. Nguyen-Xuan, T. Rabczuk, S. Bordas, and J. F. Debongnie, A smoothed finite element method for plate analysis, Computer methods in Applied Mechanics and Engineering 197 (2008) 1184-1203.

[15] M. A. Puso, J. S. Chen, E. Zywicz, and W. Elmer, Meshfree and finite element nodal integration methods, International Journal for Numerical Methods in Engineering 74 (2008) 416-446.

[16] M. A. Puso and J. Solberg, A stabilized nodally integrated tetrahedral, International Journal for Numerical Methods in Engineering 67 (2006) 841-867.

[17] J.W. Yoo, B. Moran, and J. S. Chen, Stabilized conforming nodal integration in the naturalelement method, International Journal for Numerical Methods in Engineering 60 (2004) 861890.

[18] J. W. Yoo, B. Moran, and J.S. Chen, Stabilized conforming nodal integration in the naturalelement method, International Journal for Numerical Methods in Engineering 60 (2004) 861890. 


\section{ỔN ĐỊNH HÓA CỦA PHƯƠNG PHÁP PHÀ̀N TỬ HỮU HẠN TRƠN DỰA TRÊN NÚT CHO BÀI TOÁN DAO ĐộNG TỰ DO}

Phương pháp phần tử hữu hạn trơn dựa trên nút (NS-FEM) được Liu và cộng sự đề xuất với mục đích nâng cao hiệu quả tính toán cho các bài toán cơ học vật rắn. Tuy nhiên, phương pháp NS-FEM ứng xử "quá mềm" và vì thế dẫn đến sự mất ổn định đối với các bài toán động học. Sự mất ổn định được thể hiện qua các kiểu năng lượng khác không giả (spurious non-zero energy modes) trong phân tích dao động tự do. Trong bài báo này, chúng tôi đưa ra một kỹ thuật ổn định hóa phương pháp phần tử hữu hạn trơn dựa trên nút (SN-FEM) mà nó ổn định (no spurious non-zero energy modes) và hiệu quả hơn phương pháp phần tử hữu hạn (FEM) truyền thống. Ba minh họa số được đưa ra để chứng tỏ độ tin cậy cao của công thức đề xuất. 